\title{
Role of In Vitro-In Vivo Correlations in Drug Development
}

Patrick Marroum

Clinical Pharmacology and Pharmacometrics, Abbvie Pharmaceuticals, North Chicago, Illinois

\section{INTRODUCTION}

0 ince the early 1950s, it was recognized that differences in dissolution and release characteristics impact in vivo systemic bioavailability resulting in therapeutic variability and in some cases toxicities and therapeutic failures (1). This resulted in considerable efforts in developing dissolution methods that can discriminate among formulations with differences in release characteristics and corresponding differences in bioavailability. On the other hand, the availability of many mathematical modeling techniques that allowed the accurate estimation of the fraction of drug released in vivo as a function of time enabled the establishment of a robust in vitro-in vivo correlation (IVIVC) that enabled the prediction of the plasma concentration-time profile of a formulation from its in vitro dissolution profile. For this reason, dissolution became a valuable tool that both the pharmaceutical industry and regulatory agencies utilize as a predictor or surrogate for bioavailability.

This review article will discuss the various considerations for the establishment, evaluation, and application of IVIVC. Cases studies will be presented on the application of IVIVC to obtain in vivo bioavailability waivers as well as setting meaningful dissolution specifications that are both clinically meaningful and assure the quality of the drug product from lot to lot and from within the same lot. The cases studies will encompass both the conventional oral modified-release formulations and specialized dosage forms. In addition, common misconceptions of IVIVCs will be discussed.

\section{LEVELS OF IVIVCS Level A Correlations}

A Level A correlation (2) is a point-to-point relationship between the in vitro dissolution and the in vivo input rate as can be seen in Figure 1. Such a relationship can be linear where the in vitro release rate and the in vivo input rate are superimposable. It is a misconception that nonlinear correlations are not predictive and therefore unacceptable to regulatory agencies. However these correlations are very uncommon.

\section{Level B Correlations}

In a Level B correlation, the mean in vitro dissolution time is compared with either the mean residence time or the mean in vivo dissolution time as seen in Figure 2. A Level B IVIVC uses the principals of statistical moment

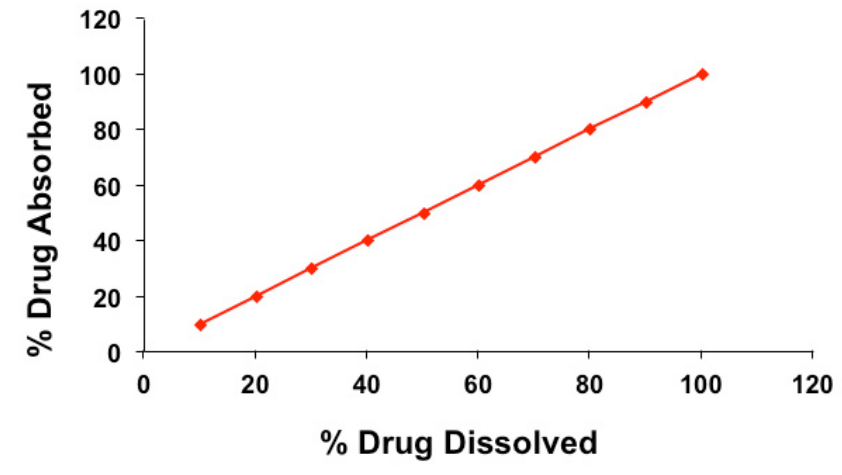

Figure 1. Level A correlation showing the point-to-point relationship between the fraction of drug absorbed and the fraction of drug dissolution.

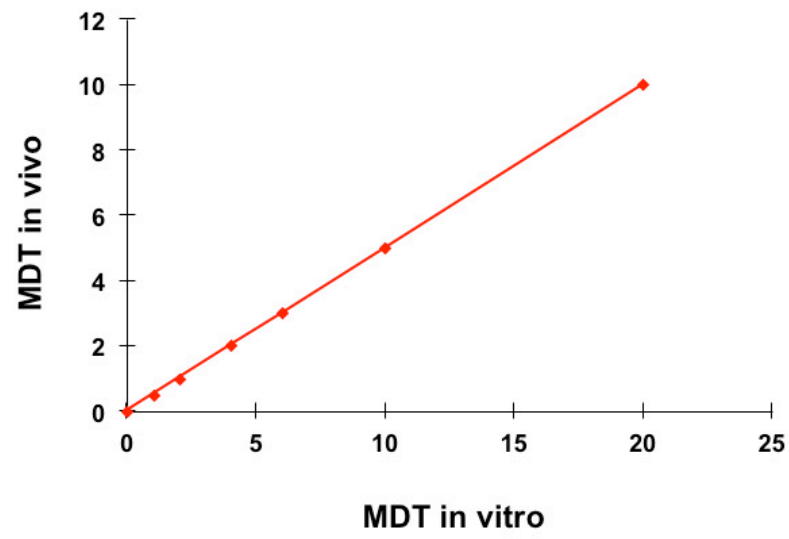

Figure 2. Level B correlation showing the relationship between the mean in vitro dissolution and the mean in vivo dissolution time.

analysis. Even though a Level B correlation uses all the in vitro and in vivo data, it is not considered a point-topoint correlation. It does not reflect the actual plasma concentration in a unique way as a number of different in vivo profiles will produce similar mean residence times. For this reason, a Level B correlation is of little value from an application point of view.

\section{Level C Correlations}

A Level C correlation establishes a relationship between a dissolution parameter such as the amount of drug released at a certain time and a pharmacokinetic parameter of interest such as the area under the curve (AUC) or the peak plasma concentration $\left(C_{\max }\right)$. Unfortunately, this type of correlation does not reflect the complete shape of the plasma concentration-time profile, which is a critical factor in defining the performance of the product. A multiple Level C correlation (Figure 3) correlates one or 
more $\mathrm{pK}$ parameters to the amount of drug dissolved at several time points of the dissolution profile. In general, if one is able to establish a multiple Level $C$ correlation, then a Level A correlation can be established and is the preferred correlation to establish. Level $\mathrm{C}$ correlations are very popular with formulators to select formulations for further development.

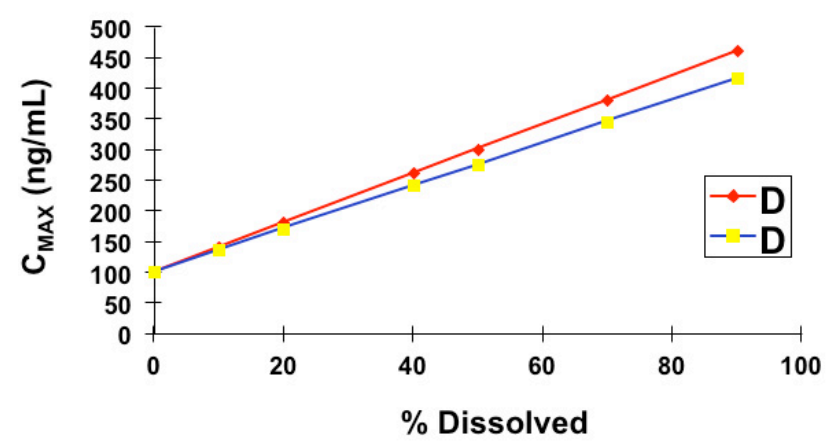

Figure 3. Level C correlation showing the relationship between the amount of drug dissolved at a certain time and the peak plasma concentration.

\section{CONSIDERATIONS IN DEVELOPING AN IVIVC In Vitro Considerations}

In many instances, the failure to establish a predictive IVIVC is because the dissolution method used is not the most discriminating method, and the refinement of the dissolution method results in a successful IVIVC. In general, there are no restrictions on the dissolution method to be used. The vast majority of successful IVIVCs utilize either USP Apparatus 1 or 2 as they are the most suitable for oral dosage forms. To preserve the discriminating ability of the method, rotational speeds in excess of compendial speeds (100-150 rpm for the basket and 50-75 rpm for the paddle) should be avoided. Aqueous media with a $\mathrm{pH}$ not exceeding 6.8 are preferred. However, for poorly soluble drugs, the addition of a solubilizing agent such as a surfactant could be appropriate. However, careful attention to the amount of surfactant should be given as adding too much surfactant will make the method less discriminating leading to the inability to develop a successful IVIVC. Nonaqueous or hydro-alcoholic solutions are generally discouraged unless all attempts with aqueous media have failed. Generating the dissolution profiles with 12 units will reduce the variability in the results. A good dissolution method to be used for the establishment of an IVIVC should not have a coefficient of variation of more than $10 \%$. It is acceptable to try different dissolution conditions in an attempt to match the in vivo profiles. However, once these conditions are identified, they should be used to validate the IVIVC and used in any application of the IVIVC.

\section{In Vivo Considerations}

A common question is whether an IVIVC model developed in animals can be used from a regulatory point of view. Since the pharmacokinetic properties of a drug tend to be somewhat different between animals and humans especially in terms of absorption, only IVIVCs developed using human data are acceptable to regulatory agencies. The only exception to this is the development of IVIVC for drug-eluting stents; it is impossible to conduct such studies in humans because of the irreversible and invasive nature of these types of products.

Other common questions are how large should be the human study and should it a statistical power comparable to a bioequivalence study. In general, the larger the variability in $\mathrm{pK}$ parameters, the larger the study should be. However, achieving the same power as a bioequivalence study is not necessary. Human studies ranging from six subjects to as many as 48 subjects were conducted to establish successful IVIVCs. Crossover studies are preferred and require fewer subjects to characterize the bioavailability of the tested formulations adequately. This does not mean that parallel designs or data from across different studies could not be used to establish the correlation.

The inclusion of an immediate-release formulation will facilitate the data analysis since it will allow a better estimate of the terminal rate constant for each subject and will enable normalization of the areas under the plasma concentration-time profile (AUC) to a common reference if differences in bioavailability exist among the different formulations. The reference product could be an intravenous solution, an aqueous solution, or an immediate-release formulation such as a tablet or a capsule.

\section{EVALUATION OF THE PREDICTABILITY OF THE IVIVC}

A crucial step in the development of an IVIVC is the determination of its applicability. This is usually achieved by evaluating how accurately and precisely it can predict the plasma concentration-time profile. The criteria for the acceptability of an IVIVC are outlined in the FDA guidance on the development, evaluation, and application of IVIVC for oral modified release formulation (2). The predictive power of the model is evaluated by the assessment of prediction error. Depending on the number of formulations used and the therapeutic index of the drug, evaluation of the internal or external predictability of the IVIVC may be warranted. The internal predictability is based on the data set that was used to develop the IVIVC. It consists of determining how well one is able to predict the plasma concentration-time profile of the formulations used to develop the correlation. The evaluation of the external predictability involves additional data sets beyond the ones used to develop the IVIVC. External predictability is warranted in the following situations: (1) the drug is considered to have a narrow therapeutic window; (2) the model was developed with fewer than three formulations with different release rates; (3) the internal predictability criteria are not met.

Ideally, the external predictability should be evaluated with a formulation with a different release rate; however, it is acceptable to use formulations with the same release characteristics as those used to develop the IVIVC. The following represent the type of formulation to be used in the evaluation of external predictability in decreasing 
order of preference:

- Formulations with different release rates.

- A formulation that was made involving a certain manufacturing change such as equipment, process, site, etc.

- Similar formulations but different lots than the ones used in the development of the IVIVC obtained from different studies within the development of the program.

An IVIVC is deemed predictive and acceptable from a regulatory point of view if the mean prediction errors for $C_{\max }$ and $A U C$ of all the formulations used to develop the correlation do not exceed $10 \%$ with none being above $15 \%$. If the mean prediction error exceeds $20 \%$, the IVIVC is considerable to be of poor predictive ability and cannot be used to support any regulatory decision. If the prediction error is greater than $10 \%$ but smaller than $20 \%$, the results are deemed to be inconclusive and further external predictability evaluations will be warranted.

In the event that the relationship between the in vitro dissolution and the in vivo absorption is dependent on the formulation used as can be seen in Figure 4, this is an indication that a consistent relationship that is predictive of the in vivo performance does not exist. This is due to the fact that depending on the formulation used, one can have different amount of drug absorbed for the same amount of drug dissolved. In contrast, a robust and consistent IVIVC would always give approximately the same slope irrespective of the formulation used (whether a slow, fast, or medium formulation is used and whether all the data is pooled). This is illustrated in Figure 5 where the slope of this predictive correlation is the same for each of the individual formulations or whether all the data is pooled together and treated as one formulation.

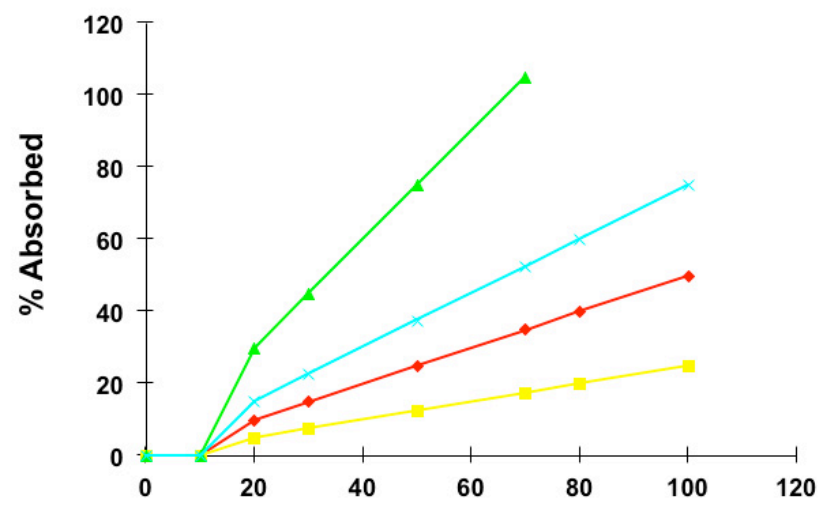

\% Dissolved

Figure 4. Poor in vitro-in vivo relationship where the slope of the relationship is dependent on the formulation used.

\section{Mean versus Individual Data}

A commonly encountered question is whether the IVIVC should be developed using individual or mean data.

The development of the IVIVC should be developed

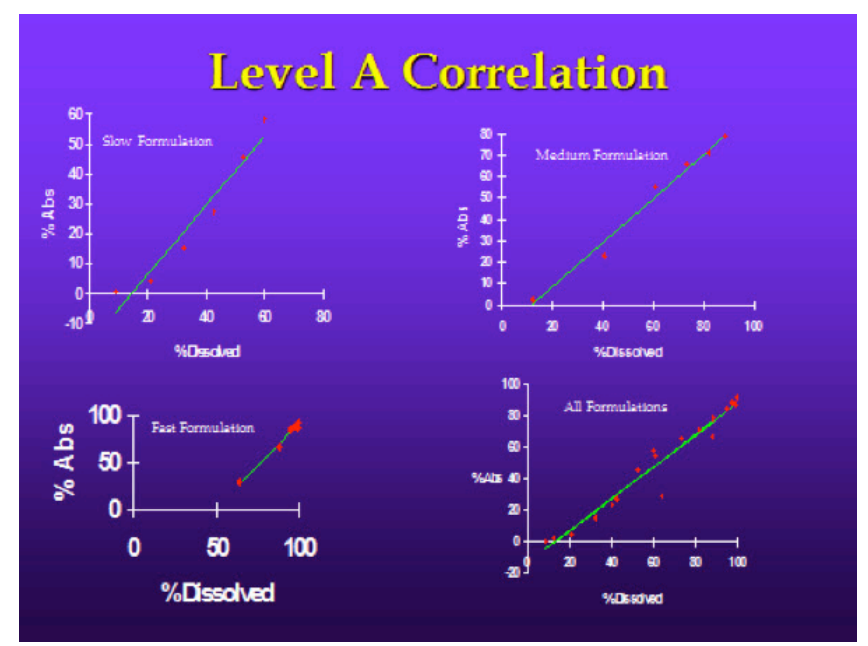

Figure 5. Predictive in vitro-in vivo correlation independent of the release rate where the slope of the relationship is independent of the formulation used.

using the individual data. In general, each plasma concentration-time profile is deconvolved to obtain the individual fraction of drug absorbed. These individual absorption profiles are then averaged and correlated with the mean dissolution profiles for the respective formulations.

However, to evaluate the predictive ability of the correlation or to predict the plasma profiles for a particular formulation from its in vitro dissolution profile, the mean plasma concentration-time profiles should be predicted because the individual dissolution for the corresponding plasma profile is not available. In addition, for comparing two formulations when individual plasma concentrations for the corresponding formulations are not available, only the mean dissolution profiles are available to predict a resulting mean plasma concentration-time profile.

\section{APPLICATIONS OF IVIVC}

Conventionally, dissolution testing is viewed as a quality control test to ensure the quality of the batch from an in vitro release point of view. However, if a predictive IVIVC is established, then dissolution could also become a surrogate for bioavailability thus allowing to evaluate the in vivo performance of a formulation without having to test it in vivo (3). Such ability will allow the granting of in vivo bioavailability waivers in instances where such studies would be required (4). Secondly, it would allow the setting of more clinically relevant dissolution specifications that will take into consideration the in vivo performance of the drug product therefore optimizing the quality of the product and maximizing the therapeutic benefit to the patient (5).

Even though it is possible to waive the requirements for in vivo bioequivalence, there are certain cases where an IVIVC cannot be used to obtain a waiver. For instance, it is not possible to gain approval for a new formulation with a different release mechanism, a higher or lower dosage strength than the range that was established to be safe 
and effective, or another sponsor's product even if the formulation has the same release mechanism.

\section{Criteria for Granting In Vivo Bioequivalence Waivers}

The FDA guidance on this topic (2) outlines the criteria for granting in vivo bioequivalence waivers. It is based on the mean predicted plasma concentration-time profile obtained from the mean in vitro dissolution. The difference in both the mean $C_{\max }$ and $A U C$ should not be more than $20 \%$ between the test and reference. It is to be noted that these criteria are somewhat different as they are not based on the confidence interval approach of the conventional criteria for bioequivalence.

\section{Dissolution Specifications}

The presence of a predictive and robust IVIVC will allow shifting the dissolution criteria from the in vitro side to the in vivo side. The upper and lower limits are not set on the basis of in vitro values obtained but on the predicted mean plasma concentration-time profile. Acceptable dissolution limits are those that do not result in more than $20 \%$ difference in predicted mean $C_{\max }$ and $A U C$ between the upper and lower limit of the specifications (usually $\pm 10 \%$ of the target or pivotal bio lot).

There is a popular belief in the industry that the use of IVIVC to set the dissolution specifications would result in very tight specifications that are restrictive from a manufacturing point of view and that will result in a considerable proportion of lots failing such specifications. It is my experience that such a belief is a misconception because more often than not, the use of the IVIVC results in specifications that are wider than the conventional $20 \%$ limit. This is because in the vast majority of cases, dissolution tends to be more sensitive than in vivo release since differences in release characteristics do not translate into differences in bioavailability. Moreover, for a wide therapeutic index drug product, it is the practice of FDA not to set dissolution specifications tighter than the $20 \%$ limit even if the prediction from the IVIVC warrants a tighter specification. However, if the drug is considered a narrow therapeutic window drug, it is possible to have specifications tighter than $20 \%$ due to clinical considerations and the need to tightly control the release characteristics to avoid potential toxicities.

Example 1: Application of a Level A Correlation to Obtain an In Vivo Bioequivalence Study for a Level 3 Manufacturing Site Change

This example is for a once-a-day, controlled-release formulation of a beta-blocker. The Level A correlation was developed using data obtained from a five-way crossover study in 10 healthy subjects where each subject was given a slow formulation, the target formulation, and a fast formulation. As a reference, each subject received an oral solution as well as an intravenous solution. The corresponding plasma concentration-time profiles are shown in Figure 6. This correlation was to grant an in vivo bioavailability waiver for a major formulation change as well as a Level 3 manufacturing site change. Subsequently, a bioequivalence study comparing the old and new formulation was conducted. The results of that study show that as predicted from the similar dissolution profiles, the two formulations were bioequivalent as shown in Figure 7 and Table 1 . This study further validates the concept that with a proper dissolution method that predicts the in vivo profile, one can make informed decisions on waiving bioequivalence studies thus reducing the amount of human bioequivalence studies (6).

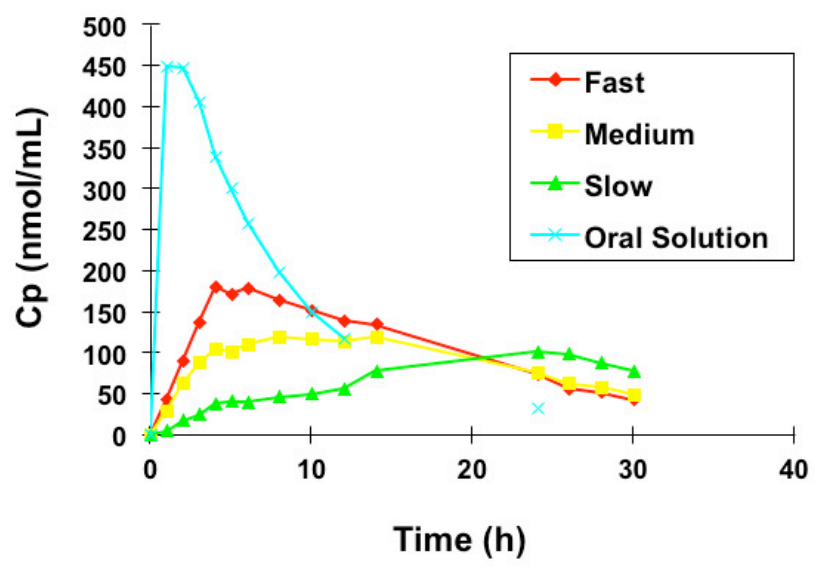

Figure 6. Plasma concentration-time profile for the formulations used to develop a Level A correlation.

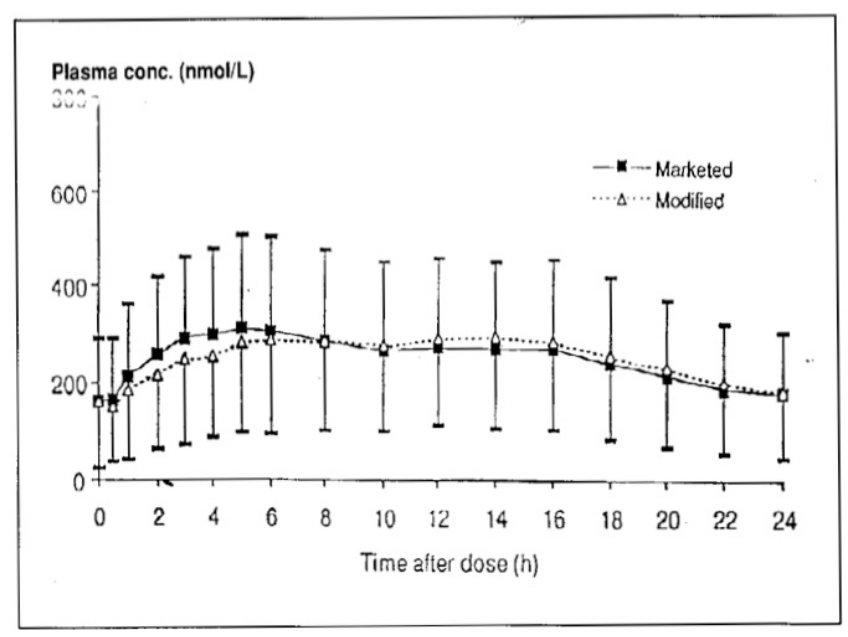

Figure 7. Plasma concentration-time profile for the old reference formulation and the new formulation (test).

Example 2: Development and Application of IVIVC for a NonOral Specialized Dosage Form

This example consists of a vaginal ring containing both etonogestrel and ethynil estradiol. It is designed to release both drugs for 21 days. As part of the development of this product, various dissolution studies were conducted to investigate the various conditions that might affect the 
Table 1. Bioequivalence Parameters Comparing the Old and New Formulations

\begin{tabular}{|l|c|c|c|}
\hline Parameter & $\begin{array}{c}\text { Modified } \\
\text { Formulation }\end{array}$ & $\begin{array}{c}\text { Original } \\
\text { Formulation }\end{array}$ & $\mathbf{9 0 \%} \mathbf{C l}$ \\
\hline$A U C$ & 6129 & 6073 & $98-108$ \\
\hline$C_{\max }$ & 316 & 327 & $93-103$ \\
\hline$C_{\min }$ & 160.7 & 165 & \\
\hline$T_{\max }$ & 10.1 & 6.14 & \\
\hline FISS & 067 & 073 & \\
\hline
\end{tabular}

FISS: Fluctuation index at steady state

release characteristics of the drug from the formulation. The results show that the release characteristics are independent of the conditions used. In addition, various prototypes with different release rates as well as drug loading were tested to investigate whether there is a relationship between the in vitro release rates and the in vivo absorption rates. Figure 8 shows the in vitro release for the various prototypes tested with the corresponding etonogestrel concentrations after five days. Figure 9 shows the linear relationship between the in vitro release rates and the in vivo absorption rates for both drug entities. Figure 10 shows the predicted versus observed plasma concentrations for both drugs, where Figure 11 shows the external predictability results. Even though the results did not strictly meet the criteria outlined in the IVIVC guidance, the IVIVC was deemed acceptable due to the complex nature of the dosage form. The sponsor subsequently changed the batch size, the manufacturing process, and the amount of non-controlling excipients. Such changes would normally require a bioequivalence study. However, since the IVIVC was deemed acceptable, these changes were approved based on comparable dissolution profiles $(7,8)$.

For specialized dosage forms where the conduct of bioequivalence studies is not as easy and straightforward as conventional oral dosage forms, the ability to evaluate the in vivo performance of such dosage forms without having to conduct in vivo studies will greatly make the development and maintenance of these products more efficient and less burdensome.

\section{IN VIVO-IN VITRO RELATIONSHIP}

It is common to establish a relationship between the in vitro dissolution and the in vivo absorption rate using just one formulation. While it is always possible to find a relationship between these two parameters, this does not constitute an IVIVC that is predictive of the in vivo performance. However, for an IVIVR to be useful, the relationship should be the same across a range of release rates and should be independent of the formulation used. However, if a quantitative relationship cannot be established, in many cases one can establish the range of dissolution rates or dissolution profiles where the

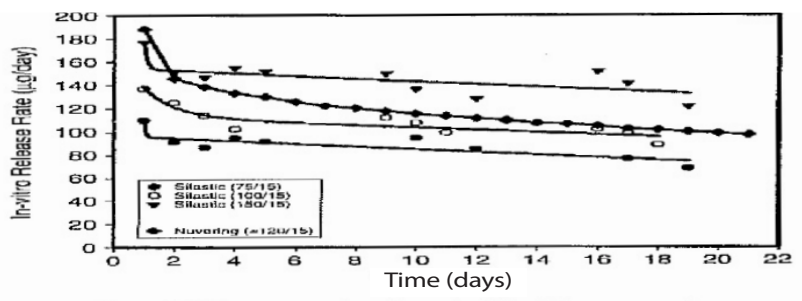

Serum ENG concentations frum the Silastiv prototypo rings were corrected in the following figure:

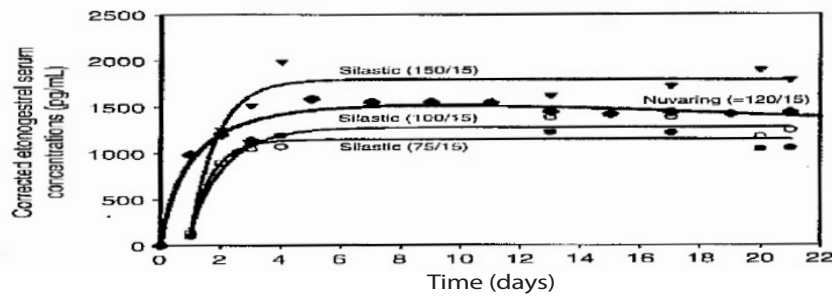

Figure 8 . In vitro release rate of the various prototypes tested with the corresponding plasma concentrations.

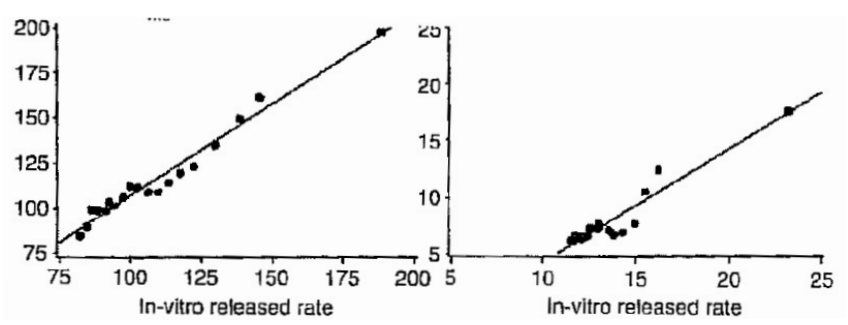

Figure 9. Relationship between the in vitro release rate and serum concentration.

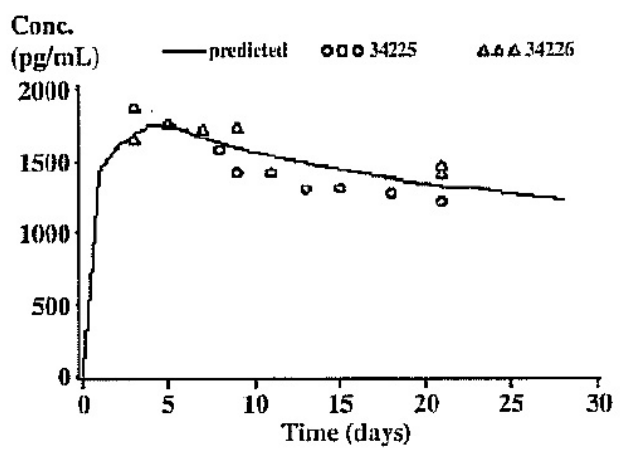

Extemal validation: Predicted vs observed in vivo concentrations for etonogestrel

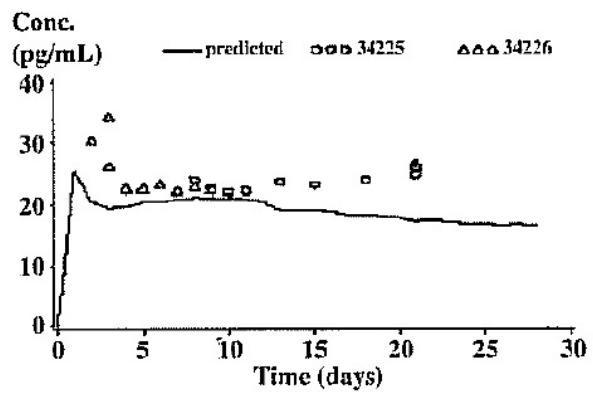

External validation: Predicted vs observed in vivo concentrations for ethinvlestradiol

Figure 10. Internal predictability showing the observed versus predicted plasma concentration-time profiles. 
formulations are bioequivalent. I recall an example where three formulations with markedly different dissolution profiles resulted in bioequivalent plasma levels. Even though with such results an IVIVC could not be established, the same results were useful to obtain wider dissolution specifications. These results would also allow the granting of in vivo bioavailability waivers as long as the resulting dissolution profile for the test formulation is within the range of dissolution profiles that were found to be bioequivalent.

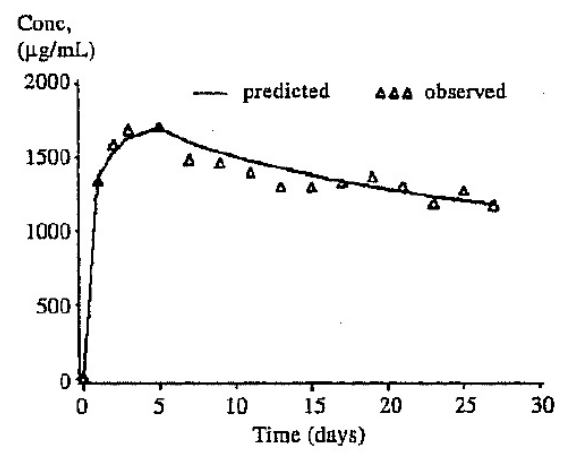

Internal validation: Predicted versus observed in-vivo serum concentrations for etonogestral

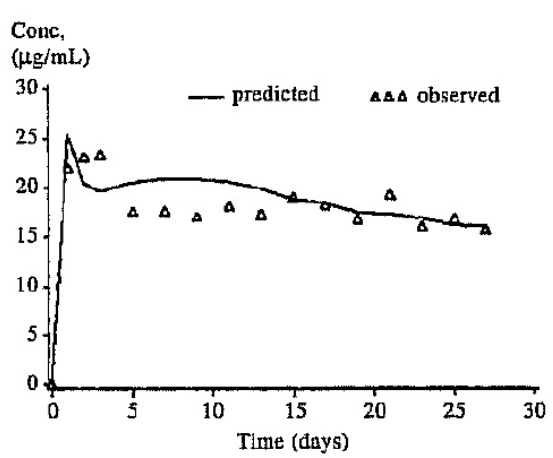

Internal validation: Predicted versus observed in-vivo serum concentrations for athinylestradiol for 1

Figure 11. External predictability showing the observed versus predicted plasma concentrations.

\section{ROLE OF IVIVC IN QUALITY BY DESIGN}

The establishment of a manufacturing design is most meaningful when it is based on in vivo considerations since such a design space will ensure that all lots/ formulations are of acceptable quality from a clinical point of view. However, since this involves the manufacturing and in vivo testing of many variants of the formulation, this task would be impossible to achieve. This is the reason that in many instances the end point for constructing the design space is in vitro dissolution. The presence of an IVIVC would therefore enable the evaluation of the in vivo outcomes without having to conduct many in vivo bioequivalence studies. The presence of an IVIVC would be optimal especially for modified-release formulations, but knowledge of the ranges of in vitro dissolution profiles that are still bioequivalent is also very useful especially for immediate-release formulations, where it is much more difficult to establish mathematical predictive correlations $(9,10)$.

\section{CONCLUSION}

The presence of an IVIVC is very beneficial during the drug development and approval process. It not only provides for an understanding of the formulations but also enables the evaluation of in vivo outcomes without having to perform costly human in vivo testing thus reducing both the cost and time it takes to develop a drug and gain approval to the market. It also would provide greater regulatory flexibility by decreasing the number of bioequivalence studies needed to approve and maintain a drug product on the market. It will also provide wider dissolution specifications that are clinically relevant. Thus, the attempt to establish IVIVC is currently more prevalent during drug development even though it is not a requirement from a regulatory point of view.

\section{ACKNOWLEDGMENT}

AbbVie contributed to the review and approval of this publication. The author is an AbbVie employee and may hold AbbVie stocks or options.

\section{REFERENCES}

1. Marroum, P. History and Evolution of the Dissolution Test. Dissolution Technol. 2014, 21 (3), 11-16. DOI: 10.14227/DT210314P11.

2. Extended Release Oral Dosage Forms: Development, Evaluation, and Application of In Vitro/In Vivo Correlations; Guidance for Industry; U.S. Department of Health and Human Services, Food and Drug Administration, Center for Drug Evaluation and Research (CDER), U.S. Government Printing Office: Washington, DC, 1997.

3. Marroum, P. J. Regulatory Examples: Dissolution Specifications and Bioequivalence Product Standards. In Scientific Foundations for Regulating Drug Product Quality; Amidon, G. L., Robinson, J. R., Williams, R. L., Eds.; AAPS Press: Alexandria, VA, 1997; pp 305-319.

4. SUPAC-MR: Modified Release Solid Oral Dosage Forms, Scale-Up and Postapproval Changes: Chemistry, Manufacturing, and Controls, In Vitro Dissolution Testing, and In Vivo Bioequivalence Documentation; Guidance for Industry; U.S. Department of Health and Human Services, Food and Drug Administration, Center for Drug Evaluation and Research (CDER), U.S. Government Printing Office: Washington, DC, 1997.

5. Marroum, P. J. Role of IVIVC in Setting Dissolution Specifications. Am. Pharm. Rev. 1999, 2, 39-42.

6. Cassella, P. Clinical Pharmacology and Biopharmaceutics Review; U.S. Department of Health and Human Services, Food and Drug Administration, Center for Drug Evaluation and Research (CDER), U.S. 
Government Printing Office: Washington, DC, April 1991.

7. Lau, S. Clinical Pharmacology and Biopharmaceutics Review; U.S. Department of Health and Human Services, Food and Drug Administration, Center for Drug Evaluation and Research (CDER), U.S. Government Printing Office: Washington, DC, December 2000.

8. Jaragula, V. Clinical Pharmacology and Biopharmaceutics Review; U.S. Department of Health and Human Services, Food and Drug Administration, Center for Drug Evaluation and Research (CDER), U.S. Government Printing Office: Washington, DC, March
2002.

9. Marroum, P. J. Clinically Relevant Dissolution Methods and Specifications. Am. Pharm. Rev. 2012, 15 (1), 3641.

10. Marroum, P. J. Dissolution and Its Relevance in Life Cycle of a Product: An FDA Perspective. Proceedings of the AAPS Annual Meeting, New Orleans, LA, November 14-18, 2010. 